\title{
On The Discrete Nature of The Gravitational Force
}

\author{
Dewey Lewis Boatmun \\ BOATMUN@sbcglobal.net
}

\begin{abstract}
The standard model of particle physics has been extremely successful in unifying the strong, weak, and electromagnetic forces through the unitary product group $\mathrm{SU}(3) \times \mathrm{SU}(2) \times \mathrm{U}(1)$; and general relativity has been equally accurate in describing gravitation. No attempt thus far, however, has managed to incorporate general relativity into the standard model-or any other model. And while no attempt will, herein, be made to accomplish that endeavor, perhaps some of the insights will constitute a helpful step in that direction. In the following pages, gravitation and electromagnetism are looked at in new and different ways.
\end{abstract}

\section{Introduction}

Waves are everywhere: water waves, sound waves, flags waving, wheels turning. Even the average temperature outside a person's home, over time, forms a sinusoidal pattern. These words, too-simple, though, they seem-are, nonetheless, being transported, processed, and communicated via electromagnetic waves, and their actual meanings and interpretations are accomplished electromagnetically.

It's an obscure fact, but wave dynamics are involved in virtually everything a person sees, or hears, or tastes, or smells, or feels, or thinks. Even the very dictates of dimensional analysis, which are required in the physical equations describing waves, are actually derived from the properties of waves themselves: wavelength (a spatial dimension) frequency (the inverse of time) and energy (the equivalent of mass); i.e., space, time, and mass.

Although all people share a natural familiarity with space, time, and mass (i.e. energy) the true meanings of these terms are, in each case, vague, and attempting to define them proves problematic, for each can be described only in terms of the other two. Energy is a function of frequency...which is a function of time... which is how one measures motion in space...which describes wavelength....which is inversely proportional to energy....and on and on...

To get quickly to the point, one need only consider the details of the random sinusoidal example below. First of all, it's obvious that the wave has a spatial length of about 20 units in some (also random) dimension. It is customary to designate this length with the Greek letter lambda $(\lambda)$, or sometimes to refer to its reduced wavelength $(\lambda=\lambda / 2 \pi)$, which is the spatial equivalent of one radian. References herein will be such that $\left(\lambda=\frac{\lambda}{2 \pi}=r_{\lambda}\right)$, where the subscript will indicate the particular wave or particle being referenced. In the present case therefore $\left(\lambda=\frac{\lambda}{2 \pi}=r_{\lambda}\right)$. 


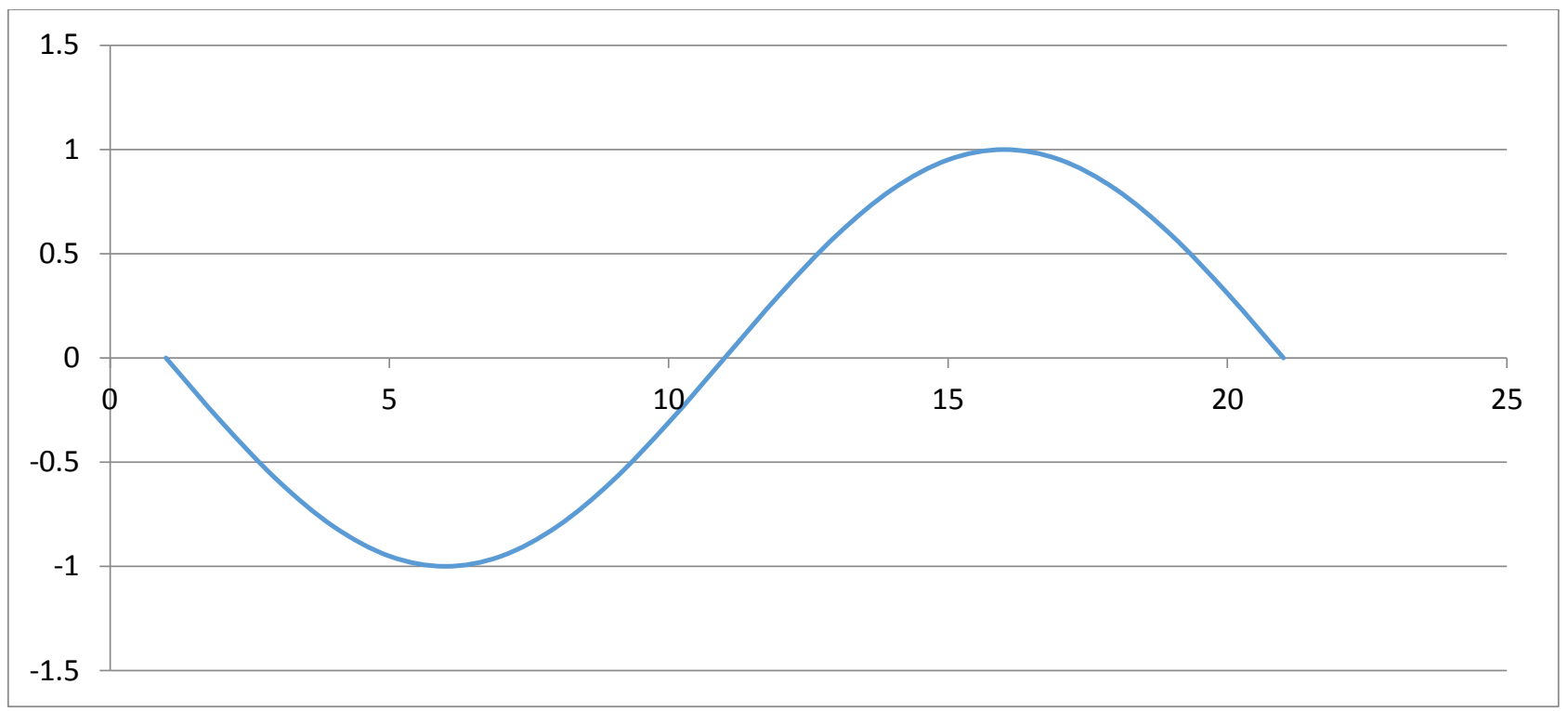

FIGURE 1

The energy of a wave increases with frequency. It takes, for instance, more energy to jump up and down 100 times in a minute that it does to do it once in a minute. Also, the engine in a car burns more gas rotating at $2,000 \mathrm{rpm}$ than it does at $200 \mathrm{rpm}$. In fact, one of the most fundamental of all constants-Planck's constantsays that the energy of any wave is proportional to its frequency, in such a way as to produce the constant $(h)$ where:

$$
E=h f
$$

and

$h=6.63 \times 10^{-34}$ joules per cycle per second

or sometimes $(\hbar)$ where:

$$
E=\hbar \omega
$$

and

$$
\hbar=1.05 \times 10^{-34} \text { joules per radian per second }
$$

where a radian is equal to

$r_{\lambda}=\lambda / 2 \pi$

and from which

$$
\left(m v r_{\lambda}=\hbar\right)
$$

for any wave or particle.

Looking at the wave again... 


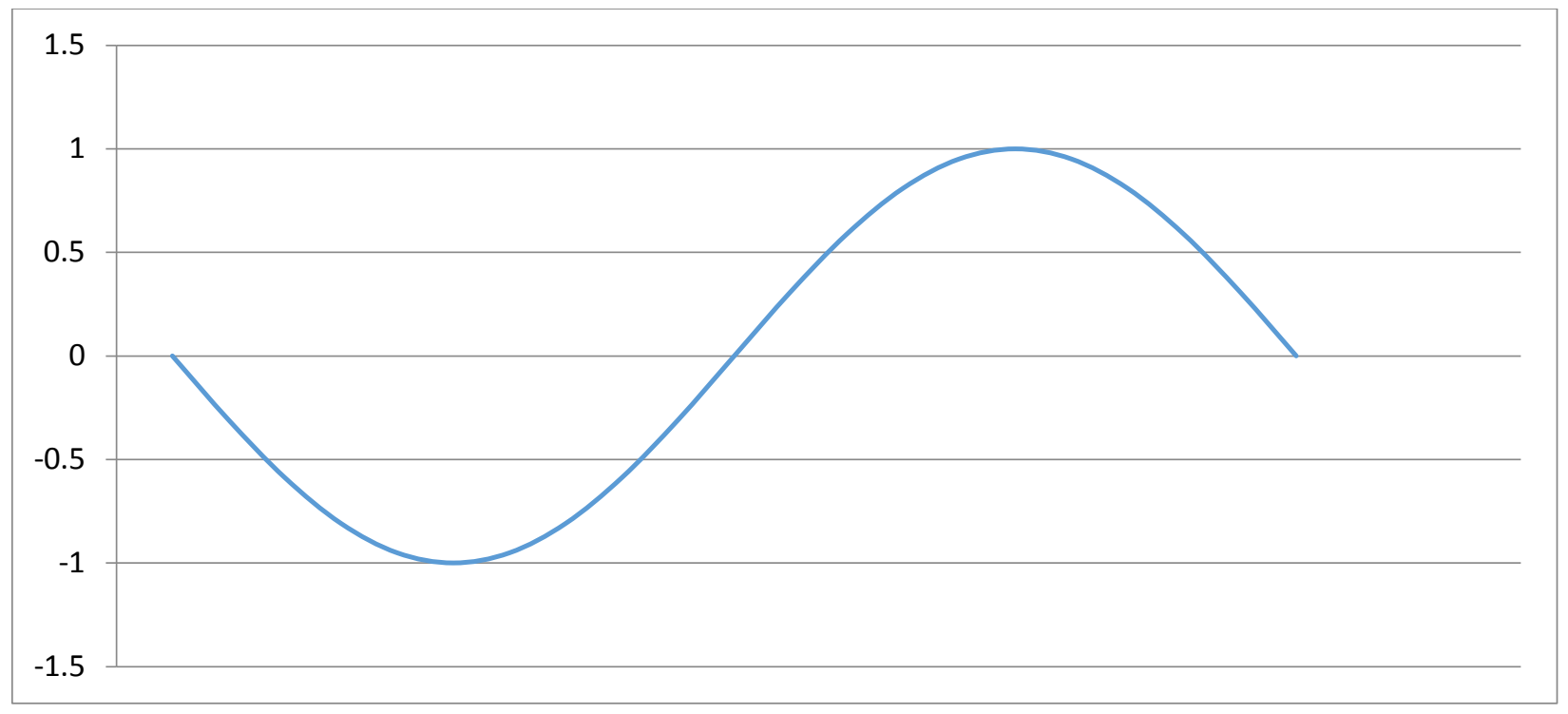

\section{FIGURE 2}

What if its frequency were to increase?

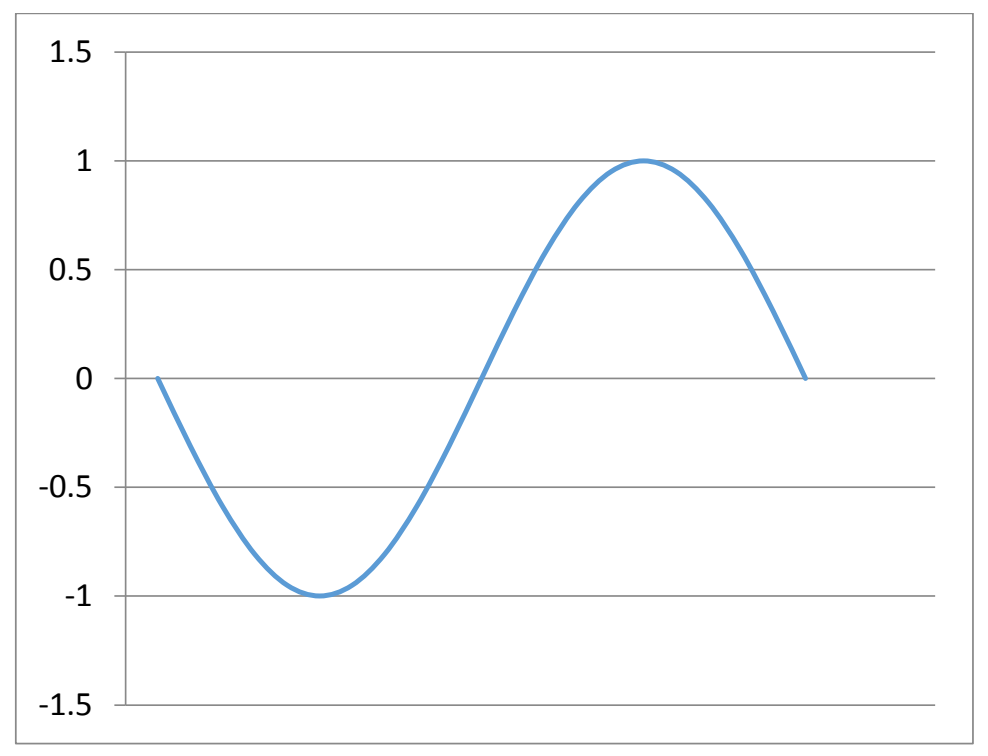

FIGURE 3

The result is clearly less space and less time...but what if its frequency were to increase even more? 


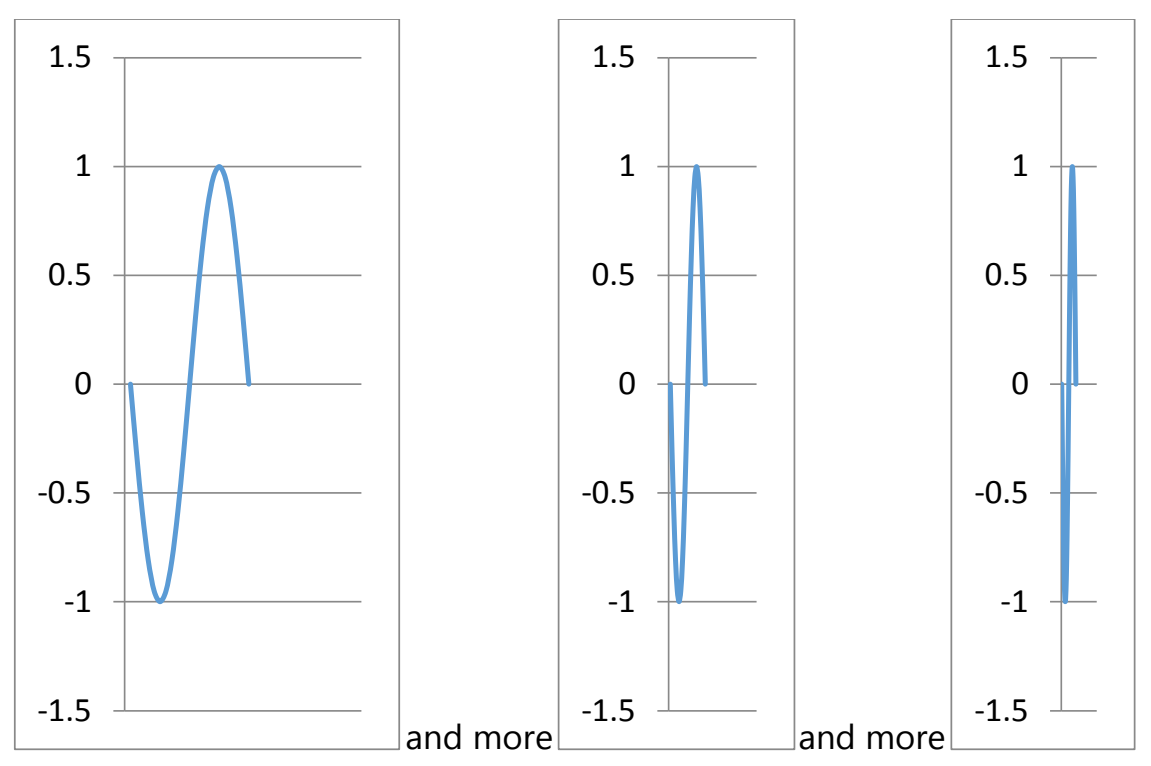

\section{FIGURE 4}

The implication is that energy (or equivalently mass) gobbles up space and time. When this is understood in the context of a wave, the most probable ensuing deliberation becomes how high the frequency could go. It would seem that it could go to infinity, and, perhaps, it can; however, curiously, all of the implied space and time is gobbled up when the frequency reaches the Planck frequency, or about $\left(1.86 \times 10^{43}\right.$ radians per second). It's as if, expressed most clearly in the last example, the width of nature's pen stroke appears to limit the oscillation's ultimate frequency. .

Nature keeps her own counsel, though. What is known for certain is that the Planck limit is the point at which She shrinks a dimension of space to nothing and stops time: a black hole. Schwarzschild was intrigued enough by her musings. It is by his formula that scientists now measure a black hole's radius $\left(r_{s}\right)$.

$$
r_{s}=\frac{2 G m}{c^{2}}
$$

And it happens that:

$$
\frac{G}{c^{2}}=\frac{r_{\text {planck }}}{m_{\text {planck }}}
$$

So that:

$$
\frac{r_{s}}{r_{\text {planck }}}=\frac{2 m}{m_{\text {planck }}}
$$

In fact, Einstein's field equations of general relativity state that:

$$
G_{\mu v}=\frac{8 \pi \mathrm{G}}{\mathrm{c}^{4}} \mathrm{~T}_{\mu v}
$$

$\left(G_{\mu v}\right)$-the Einstein tensor-represents space-time curvature, and $\left(T_{\mu v}\right)$-the stress-energy tensor-represents energy density, so that: 


$$
\frac{c^{4}}{G}=(8 \pi) \frac{T_{\mu v}}{G_{\mu v}}
$$

The left side of this equation is called the Planck force. The Planck force is a constant force which is equal to $\left(\frac{\left(m_{\text {planck }}\right) c^{2}}{\left(r_{\text {planck }}\right)}\right)$ and has the tremendous value of $1.21 \times 10^{44}$ newtons. The equation as a whole implies that the Planck force defines the relationship between energy density and space-time curvature.

Closer scrutiny yields supporting confirmation of this implication. The universe has been expanding for 13.8 billion years or so. That's about ( $t=4.36 \times 10^{17}$ seconds). Also, 13.8 billion light years corresponds to a distance of about ( $d=1.31 \times 10^{26}$ meters). The aforementioned Planck force, when exerted over $1.31 \times 10^{26}$ meters would result in an incredible amount of energy ( $E=1.58 \times 10^{70}$ joules). The mass equivalent of this energy would equal ( $m=\frac{E}{c^{2}}=1.76 \times 10^{53}$ kilograms).

It would help if one were to imagine a cube expanding in three dimensions, wherein the rate of change in the expansion (cubic meters per second per second) were dependent on the amount of energy the cube contained. The mass equivalent of that energy will be used in the following example to derive a constant $(K)$, such that $\left(K=\frac{d^{3}}{m t^{2}}\right)$. Plugging-in the numbers:

$$
K=\frac{\left(1.31 \times 10^{26} \mathrm{~m}\right)^{3}}{\left(1.76 \times 10^{53} \mathrm{~kg}\right)\left(4.36 \times 10^{17} \mathrm{sec}\right)^{2}}=6.67 \times 10^{-11} \frac{\mathrm{m}^{3}}{\mathrm{~kg} \cdot \mathrm{sec}^{2}}=6.67 \times 10^{-11} \frac{\mathrm{n} \cdot \mathrm{m}^{2}}{\mathrm{~kg}^{2}}
$$

which, of course, is Isaac Newton's gravitational constant.

To return, now, to a discussion of the Planck wave itself: If one were to discover the effect of any other wave (or group of waves) on space-time curvature, he need only compare it to the Planck wave.

For instance, let's take a giant wave of some sort, with a wavelength of $\left(4.02 \times 10^{7}\right.$ meters $)$ and an energy of $\left(5.39 \times 10^{41}\right.$ joules $)$. It sounds dimensionally enormous, and it is, but it pales in comparison with the Planck wave. In any case, $\left(5.39 \times 10^{41}\right.$ joules $)$ converts to a mass equivalent of $(6.0 \times$ $10^{24}$ kilograms) - the earth's mass - and ( $4.02 \times 10^{7}$ meters $)$ divided by $2 \pi$ is $\left(6.4 \times 10^{6}\right.$ meters $)$-the earth's radius. Comparing it to the Planck wave:

1

$$
\frac{\frac{m}{r}}{\frac{m_{\text {planck }}}{r_{\text {planck }}}}=\frac{\frac{m_{\text {earth }}}{r_{\text {earth }}}}{\frac{m_{\text {planck }}}{r_{\text {planck }}}}=\frac{\frac{6.0 \times 10^{24} \mathrm{~kg}}{6.4 \times 10^{6}}}{\frac{2.18 \times 10^{-8} \mathrm{~kg}}{1.62 \times 10^{-35} \mathrm{~m}}}=\frac{v^{2}}{c^{2}}
$$

Solving for $\left(v^{2}\right)$ and dividing by $\left(r_{\text {earth }}\right)$ produces a familiar number: the gravitational acceleration at the earth's surface- $-9.8 \mathrm{~m} / \mathrm{sec}^{2}$.

Because:

$$
\frac{m_{\text {planck }}}{r_{\text {planck }}}=\frac{c^{2}}{G}
$$

the above equation simply says

$$
\frac{G m_{\text {earth }}}{r_{\text {earth }}^{2}}=\frac{v^{2}}{r}
$$

which is simply Newton's gravitational acceleration equation.

In fact, for any two masses $\left(\mathrm{m}_{1}\right.$ and $\left.\mathrm{m}_{2}\right)$, where: 


$$
\frac{m_{1} v^{2}}{r}=\frac{G m_{1} m_{2}}{r^{2}}
$$

it's a fact that:

$$
\frac{m_{2} u^{2}}{r}=\frac{c^{4}}{G}
$$

where $u$ is the gravitational phase velocity $\left(u=\frac{c^{2}}{v}\right)$, and $\left(\frac{c^{4}}{G}\right)$ is, of course, the Planck force. For instance, in the example above, if the gravitational acceleration $\left(\frac{v^{2}}{r}\right)$ at the earth's surface is $9.8 \mathrm{~m} / \mathrm{sec}^{2}$, and $\mathrm{m}_{\text {earth }}$ and $\mathrm{r}_{\text {earth }}$ are as shown above, then $(\mathrm{v}=7,920 \mathrm{~m} / \mathrm{sec}),\left(\mathrm{u}=\frac{\mathrm{c}^{2}}{\mathrm{v}}=1.13 \times 10^{13} \mathrm{~m} / \mathrm{sec}\right)$, and:

$$
\frac{m_{\text {earth }} u^{2}}{r}=\frac{c^{4}}{G}=1.21 \times 10^{44} \text { newtons }
$$

While the Planck wave is unique in its $\left(\frac{m}{r}\right)$ ratio, it is not so in its $(m \cdot r)$ product. Any wave which moves at the speed of light is usually called a "light wave", for obvious reasons. Like any other oscillation, the product of its momentum and reduced wavelength must be such that:

$$
m v r_{\lambda}=\hbar
$$

or some integer multiple thereof. And when $(v=c)$, that becomes:

Because $c$ and $\hbar$ are both constants here, so too must the product $\left(m \cdot r_{\lambda}\right)$ be, regardless of the frequency. Again, the Planck wave is not unique in this regard, but it's about the only wave that has a name, so, for the purpose of simplicity, its dimensions will be used in the following example.

$$
\left(m_{\text {planck }}\right)\left(r_{\text {planck }}\right)=\left(2.18 \times 10^{-8} \mathrm{~kg}\right)\left(1.62 \times 10^{-35} \mathrm{~m}\right)=3.53 \times 10^{-43} \mathrm{~kg} \cdot \mathrm{m}
$$

Obviously, the same would be true for a light wave of any frequency. It turns out that:

$$
3.53 \times 10^{-43} \mathrm{~kg} \cdot \mathrm{m}=\frac{10^{-7} e^{2}}{\alpha}
$$

where $e$ is coulombs per unit charge, and $\alpha$ is the fine structure constant (which is dimensionless). Actually, this is not surprising in view of the nature of a "light wave". In fact, the information contained in any electromagnetic exchange, consists of some integer multiple of this.

Since a particle, such as an electron, has a Compton wavelength, such that:

$$
\frac{\lambda}{2 \pi}=\frac{\hbar}{m c}
$$

i.e.

$$
m c r_{\lambda}=\hbar,
$$

and since $c$ and $\hbar$ are constants, it follows, again, that this product $\left(m \cdot r_{\lambda}\right)$ is also a constant, and:

$$
m=\frac{10^{-7} e^{2}}{r_{m} \alpha}
$$

where $r_{m}$ is the reduced wavelength associated with that particular mass. It can be seen that the dimension for the right hand side of the equation is, in fact:

$$
\frac{\text { joules }}{c^{2}}=k g
$$


Meanwhile the gravitational force for a ground state hydrogen atom is such that:

$$
\left(\frac{G m_{\text {elec }} m_{\text {prot }}}{\alpha_{0}{ }^{2}}\right)=\left(\frac{\alpha}{1} \frac{m_{\text {elec }} G m_{\text {prot }}}{\alpha_{0}{ }^{2}} \frac{1}{\alpha}\right)=\frac{10^{-7} e^{2}}{r_{\text {elec }}} \frac{G m_{\text {prot }}}{\alpha_{0}{ }^{2}} \frac{1}{\alpha}=\frac{c^{2} X 10^{-7} e^{2}}{\alpha_{0}{ }^{2}} \frac{G m_{\text {prot }}}{r_{\text {elec }} \alpha c^{2}}
$$

where $m_{\text {elec }}$ is electron mass, $m_{\text {prot }}$ is proton mass, $\alpha$ is the fine structure constant, $\alpha_{0}$ is the Bohr radius, $\left(\frac{c^{2} \times 10^{-7} e^{2}}{\alpha_{0}{ }^{2}}\right)$ is the electrostatic force, and the factor in blue is a scalar composed of five well-known universal constants, whose values here account for the dimensionless ratio of the strengths of the electrostatic force to the gravitational force.

$$
\frac{G m_{\text {prot }}}{r_{\text {elec }} \alpha c^{2}}=\left(2.27 \times 10^{39}\right)^{-1}
$$

The factor $\left(\frac{G m_{\text {prot }}}{r_{\text {elec }} c^{2}}\right)$ is dimensionless, as is $(\alpha)$, which is the electromagnetic coupling constant, and which is equal to $\left(\frac{v_{\alpha_{0}}}{c}\right)$, where $\left(v_{\alpha_{0}}\right)$ is the electron group velocity in the present example $\left(v_{\alpha_{0}}=2.19 \times\right.$ $\left.10^{6} \mathrm{~m} / \mathrm{sec}\right)$. $\left(\frac{G m_{\text {prot }}}{r_{\text {elec }} c^{2} \alpha}\right)$ therefore, defines the ratio of two coupling constants, and both will be considered in the same format (i.e. $\frac{v}{c}$ ). The factor in blue is therefore,

$$
\frac{G m_{\text {prot }}}{r_{\text {elec }} \alpha c^{2}}=\left(\frac{v^{2}}{c^{2}} \frac{1}{\alpha}\right)=\left(\frac{v_{?}}{c} \frac{1}{\alpha}\right)=\left(\frac{v_{?}}{c} \frac{c}{v_{\alpha_{0}}}\right)=\left(2.27 \times 10^{39}\right)^{-1}
$$

In which case, the relative strengths of the gravitational and electrostatic forces are as follows:

$$
\frac{F_{G}}{F_{e}}=\frac{G m_{\text {prot }}}{r_{\text {elec }} c^{2} \alpha}=\left(\frac{v_{?}}{c} \frac{c}{v_{\alpha_{0}}}\right)=\left(2.27 \times 10^{39}\right)^{-1}
$$

Solving for $\left(v_{?}\right)$, in the gravitational case, results in $\left(9.63 \times 10^{-34} \mathrm{~m} / \mathrm{sec}\right)$. This result will be important later on. It should be noted, at this point, that because

$$
\frac{c^{2}}{G}=\frac{m_{\text {planck }}}{r_{\text {planck }}}
$$

the factor in blue is also equal to

$$
\frac{\frac{m_{\text {prot }}}{r_{\text {elec }}} \frac{1}{\alpha}}{\frac{m_{\text {planck }}}{r_{\text {planck }}}}
$$

This will also be important later on.

Next, it should be noted that, after also converting $\left(m_{\text {prot }}\right)$, in (Eq 2$)$ to

$$
m_{\text {prot }}=\frac{10^{-7} e^{2}}{r_{\text {prot }} \alpha}
$$

and $(G)$ to

$$
G=\frac{r_{\text {planck }} c^{2}}{m_{\text {planck }}}
$$

the equation becomes

$$
\frac{G m_{\text {elec }} m_{\text {prot }}}{\alpha_{0}^{2}}=\frac{\mathrm{c}^{2} \times\left(\left(10^{-7}( \pm e)( \pm e) \mathrm{kg} \cdot \mathrm{m}\right)^{2}\right.}{\alpha_{0}^{2}} \cdot \frac{1}{r_{\text {elec }} r_{\text {prot }}} \cdot \frac{r_{\text {planck }}}{m_{\text {planck }}} \cdot \frac{1}{\alpha^{2}}
$$


This seems messy. Clearly, though, regardless of the polarity assigned to either of the individual charges, the resulting gravitational force is always unidirectional.

Rearranging (Eq. 2):

$$
\begin{aligned}
& \frac{c^{2} X 10^{-7} e^{2}}{\alpha^{2}}=\frac{\left(G m_{\text {elec }} m_{\text {prot }}\right)\left(r_{\text {elec }} \alpha c^{2}\right)}{\left(\alpha_{0}^{2}\right)\left(G m_{\text {prot }}\right)} \\
& =\frac{\left(m_{\text {elec }} c^{2}\right)\left(r_{\text {elec }} \alpha\right)}{\alpha_{0}^{2}} \\
& =\frac{\left(m_{\text {elec }} c r_{\text {elec }}\right)(c)(\alpha)}{\alpha_{0}^{2}} \\
& =\frac{(\hbar c)(\alpha)}{\alpha_{0}^{2}}
\end{aligned}
$$

So that

$$
\frac{c^{2} \times 10^{-7} e^{2}}{\alpha_{0}^{2}}=\frac{1}{4 \pi \epsilon} \frac{e^{2}}{\alpha_{0}^{2}}=\frac{(\hbar c)(\alpha)}{\alpha_{0}^{2}}=\frac{(\hbar v)}{\alpha_{0}^{2}}
$$

where $(v)$ is the hydrogen ground state electron group velocity $\left(2.19 \times 10^{6} \mathrm{~m} / \mathrm{sec}\right)$.

And, from (Eq. 4):

$$
\alpha=\frac{1 e^{2}}{4 \pi \epsilon \hbar c}
$$

which is, of course, one of the many equations defining $(\alpha)$.

Actually, a short detour is appropriate at this point to discuss one aspect of special relativity. Special relativity says that:

$$
\frac{l}{l_{0}}=\frac{t}{t_{0}}=\sqrt{1-\frac{v^{2}}{c^{2}}}
$$

where $l_{0}$ is inertial frame length, $t_{0}$ is inertial frame time, and $l$ and $t$ are proper length and proper time, respectively. Therefore, (using length as an example):

$$
\frac{l^{2}}{l_{0}^{2}}+\frac{v^{2}}{c^{2}}=1
$$

Now, using arbitrary dimensions of length and time, one could, for instance, make $l_{0}$ equal to one light second and $c$ equal to one light second per second, so that the above (dimensionless) equation would become

$$
\frac{l^{2}}{1}+\frac{v^{2}}{1}=1
$$

This defines a unit circle that would look something like this, where the hypotenuse is the circle's radius. 


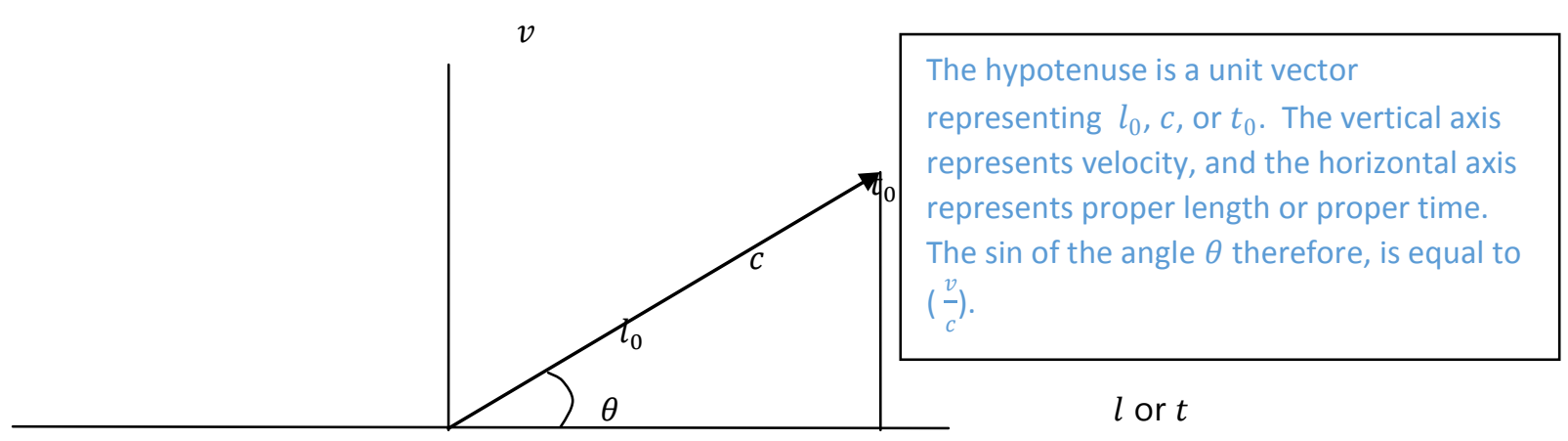

FIGURE 5

In the example of a ground state hydrogen atom, therefore, the sine of the angle $\theta$ would be

$$
\frac{2.19 \times 10^{6} \mathrm{~m} / \mathrm{sec}}{3.00 \times 10^{8} \mathrm{~m} / \mathrm{sec}}=\alpha \sim \frac{1}{137}
$$

the fine structure constant.

As previously discussed, $\left(10^{-7} e^{2}=(m)\left(r_{m}\right)(\alpha)\right.$ ), and $\left(u v=c^{2}\right)$, where $u$ is phase velocity and $v$ is group velocity, so that, for our ground state hydrogen atom, where $\left(v=2.19 \times 10^{6} \mathrm{~m} / \mathrm{sec}\right)$ and $\left(u=\frac{c^{2}}{v}=\right.$ $4.11 \times 10^{10} \mathrm{~m} / \mathrm{sec}$ ):

$$
\frac{c^{2} \times 10^{-7}(e)(e)}{\alpha_{0}^{2}}=\frac{\left(m_{\text {elec }} v\right)\left(r_{\text {elec }}\right)(\sin \theta)(u)}{\alpha_{0}^{2}}
$$

Rearranging,

$$
\begin{gathered}
\frac{c^{2} \times 10^{-7}(q)(q)}{\alpha_{0}^{2}}=\frac{\left(m_{\text {elec }} u\right)\left(r_{\text {elec }}\right) \sin \theta(v)}{\alpha_{0}^{2}} \\
(u)(\sin \theta)=\left(4.11 \times 10^{10} \mathrm{~m} / \mathrm{sec}\right)(\alpha)=c
\end{gathered}
$$

So that

$$
\frac{c^{2} \times 10^{-7}(q)(q)}{\alpha_{0}^{2}}=\frac{c^{2} \times 10^{-7}(+1) e(-1) e}{\alpha_{0}^{2}}=\frac{(m)(c)(r)(v)}{\alpha_{0}^{2}}=\frac{(-1) \hbar v}{\alpha_{0}^{2}}=\frac{(-1)(\hbar c)(\alpha)}{\alpha_{0}^{2}}
$$

as in (Eq. 4).

A few additional rearrangements will allow for a smoother transition into the next subject.

$$
\begin{gathered}
\frac{c^{2} \times 10^{-7}(e)(e)}{\alpha_{0}^{2}}=\frac{\left(\left(m_{\text {elec }} v\right) \times\left(r_{\text {elec }}\right)\right)(u)}{\alpha_{0}^{2}}=\frac{\left(m_{\text {elec }} u\right)\left(r_{\text {elec }}\right) \sin \theta(v)}{\alpha_{0}^{2}} \\
=\frac{10^{-7} e^{2}}{\alpha} \cdot \frac{(u \cdot \alpha \cdot v)}{\alpha_{0}^{2}}=\frac{10^{-7} e^{2}}{1} \cdot \frac{(u \cdot v)}{\alpha_{0}^{2}} \cdot \frac{\alpha}{\alpha}=\frac{10^{-7}(u q)(q v) \sin \left(\frac{\pi}{2}\right)}{\alpha_{0}^{2}} \\
=\frac{\left(4 \pi \times 10^{-7}\right)(u q) \sin \frac{\pi}{2}(q v)}{4 \pi \alpha_{0}^{2}}=\frac{\mu(i d l) \sin \frac{\pi}{2}(q v)}{4 \pi \alpha_{0}^{2}}=(B) \times(q v)
\end{gathered}
$$

which, of course, relates an electrical force to a magnetic force. Notice, incidentally, that $u q=i d l$. Using a ground state hydrogen atom again, as an example, where $\mathrm{v}=2.19 \times 10^{6} \mathrm{~m} / \mathrm{sec}$ and 
$\mathrm{u}=\frac{\mathrm{c}^{2}}{\mathrm{v}}=4.11 \times 10^{10} \mathrm{~m} / \mathrm{sec}:$

$$
\frac{c^{2} X 10^{-7} q}{r^{2}}=\frac{10^{-7}([u] \cdot q) \cdot([v]) \sin \frac{\pi}{2}}{r^{2}} \text { and } \frac{c^{2} X 10^{-7} q q}{r^{2}}=\frac{10^{-7}([u] \cdot q) \cdot(q \cdot[v]) \sin \frac{\pi}{2}}{r^{2}}=\frac{\hbar c(\alpha)}{r^{2}}
$$

or:

$$
\frac{10^{-7}([u] \cdot q) \cdot(q \cdot[v]) \sin \frac{\pi}{2}}{r^{2}}=\frac{\hbar c(\alpha)}{r^{2}}=\frac{\hbar\left(\frac{3.0 \times 10^{8} \mathrm{~m} / \mathrm{sec}}{137}\right)}{r^{2}}=\frac{\hbar\left(2.19 \times 10^{6} \mathrm{~m} / \mathrm{sec}\right)}{r^{2}}=\frac{\hbar(v)}{r^{2}}
$$

so that :

$$
B \times(q v)=\frac{(\hbar)(v)}{r^{2}} \text { and } \frac{\left(\left(\mu i d l \sin \left(\frac{\pi}{2}\right)(q)\right)\right.}{4 \pi}=\hbar
$$

Let's see:

$$
\begin{aligned}
\left(10^{-7}(u)(q) \sin \frac{\pi}{2}\right)(q)= & \left(1 0 ^ { - 7 } \left(\left(4,11 \times 10^{10} \mathrm{~m} / \mathrm{sec}\right) \cdot\left(1.6 \times 10^{-19}\right)( \pm 1) \cdot\left(1.6 \times 10^{-19}\right)( \pm 1)\right.\right. \\
= & ( \pm 1) 1.05 \times 10^{-34} \mathrm{joule} \cdot \sec =( \pm 1) \hbar
\end{aligned}
$$

Of course, because the ground state is a quantum state, this is not necessarily surprising. It is, in fact, the intersection of an oscillating electric field with its oscillating magnetic field, the two of which are orthogonal, leading to a "double" ( $\mathrm{h}$ ):

$$
\left(10^{-7}(u)(q) \sin \frac{\pi}{2}\right)(q)=\hbar
$$

and

$\left(m_{\text {elec }}\right)\left(v_{\text {elec }}\right)\left(\alpha_{0}\right)=\hbar$

In fact, ignoring, for now, the specifics from which it has been derived, one equation for the electrostatic force, in this "particular" instance, is as follows:

$$
\frac{c^{2} \times 10^{-7} q_{\text {elec }} q_{\text {prot }}}{\alpha_{0}^{2}}=\frac{(-1) \hbar(+1) \hbar}{m_{\text {elec }} \alpha_{0}^{3}}
$$

and in "every" instance is:

$$
\frac{c^{2} \times 10^{-7} q q}{r^{2}}=\frac{( \pm n) \hbar( \pm n) \hbar}{r^{2} m_{\text {elec }} a_{0}}
$$

Going on now, because:

$$
(m)(r)=\frac{10^{-7} e^{2}}{\alpha}
$$

or, stated thus,

$$
(m)(\alpha)(r)=10^{-7} e^{2}
$$

and since

$$
\frac{c^{2} X 10^{-7} q q}{r^{2}}=\frac{10^{-7}([u] \cdot q) \cdot(q \cdot[v]) \sin \frac{\pi}{2}}{r^{2}}
$$

this simply means 


$$
\left(\frac{c^{2} \times 10^{-7} e^{2}}{r^{2}}\right)=F_{e}=\left(\frac{\left(m(u v) r_{m}\right)(\alpha)}{r^{2}}\right)=\left(\frac{\left(m_{\text {planck }}(u v) r_{\text {planck }}\right)(\alpha)}{r^{2}}\right)
$$

Finally, plugging the result of (Eq. 5) into (Eq. 2), and keeping in mind (Eq. 3):

$\left(\frac{\left(m(u v) r_{m}\right)(\alpha)}{r^{2}}\right) \cdot \frac{\frac{m_{\text {prot }}}{r_{\text {elec }}} \frac{1}{\alpha}}{\frac{m_{\text {planck }}}{r_{\text {planck }}}}=F_{G}=\frac{G m_{\text {elec }} m_{\text {prot }}}{r^{2}}$

And, because $\left(m \cdot r_{m}\right)$ is always equal to $\left(m_{\text {planck }} \cdot r_{\text {planck }}\right)$;

$$
\frac{(u v)\left(r_{\text {planck }}^{2}\right)}{r^{2}} \cdot \frac{m_{\text {prot }}}{r_{\text {elec }}}=\left(\left(\frac{\left(r_{\text {planc }}\right)}{r_{\text {elec }}}\right) \cdot \frac{\left(m_{\text {prot }}(u v) r_{\text {planck }}\right.}{r^{2}}\right)=\frac{G m_{\text {elec }} m_{\text {prot }}}{r^{2}}
$$

Actually, $\left(\frac{m_{\text {prot }}}{r_{\text {elec }}}\right)$ is equal to $\left(\frac{m_{\text {elec }}}{r_{\text {prot }}}\right)$, so that the equation above is equally valid when written as:

$$
\left(\frac{\left(r_{\text {planc }}\right)}{r_{\text {prot }}}\right) \cdot \frac{\left(m_{\text {elec }}(u v) r_{\text {planck }}\right.}{r^{2}}=\frac{G m_{\text {prot }} m_{\text {elec }}}{r^{2}}
$$

The dimensionless factor $\left(\frac{r_{\text {planck }}}{r_{\text {prot }}}\right)$ shall hereafter be referred to as $(\beta)$, and will vary, depending on the $\left(r_{\lambda}\right)$ value of the denominator. The above equation, then, would appear as follows:

$$
\frac{G m_{\text {prot }} m_{\text {elec }}}{r^{2}}=\frac{\left(m_{\text {elec }}(u v) r_{\text {planck }}(\beta)\right.}{r^{2}}
$$

Therefore (assuming unit electrical charges for $F_{e}$, and ignoring polarity):

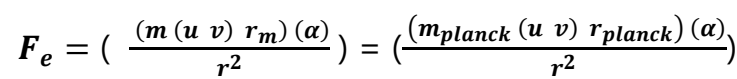

and

$$
\boldsymbol{F}_{G}=\left(\frac{\left(\boldsymbol{m}(\boldsymbol{u} v) r_{\text {planck }}\right)(\boldsymbol{\beta})}{r^{2}}\right)
$$

Or, applying (Eq. 9) in a more familiar context, using the earth, again, as an example where, $\left(r_{\text {earth }}=\frac{\hbar}{\left(m_{\text {earth }}\right) \mathrm{C}}=5.86 \times 10^{-68} \mathrm{~m}\right),(r)$ represents the earth's "actual" physical radius of $\left(6.4 \times 10^{6} \mathrm{~m}\right)$, $\left(\beta=\frac{r_{\text {planck }}}{r_{\text {earth }}}=\left(\frac{\left(1.62 \times 10^{-35} \mathrm{~m}\right)}{\left(5.86 \times 10^{-68} \mathrm{~m}\right)}\right)\right),\left(m_{\text {earth }}=6.0 \times 10^{24} \mathrm{~kg}\right)$, and $\left((u v)=c^{2}\right)$, the numbers speak for themselves.

$$
\begin{gathered}
\text { Acceleration }=\frac{F_{G}}{m}=\left(\frac{\left((\boldsymbol{u} v) \boldsymbol{r}_{\text {planck }}\right)(\boldsymbol{\beta})}{\boldsymbol{r}^{2}}\right) \\
=\frac{\left(c^{2}\right)\left(1.62 \times 10^{-35} \mathrm{~m}\right)}{\left(6.4 \times 10^{6} \mathrm{~m}\right)^{2}} \cdot\left(\frac{\left(1.62 \times 10^{-35} \mathrm{~m}\right)}{\left(5.86 \times 10^{-68} \mathrm{~m}\right)}\right)=\frac{G m_{\text {earth }}}{r^{2}}=9.8 \mathrm{~m} / \mathrm{sec}^{2}
\end{gathered}
$$

It is possible, to express the dimensionless factor $\left(\beta=\frac{r_{\text {planck }}}{r_{\text {elec }}}\right)$, in the same format as the fine structure constant $\left(\alpha=\frac{v}{c}\right)$. Of course, there will be different versions of this factor, depending on which gravitational mass is referenced in its denominator. For instance, when considering the gravitational force between the electron and the proton, as in (Eq 6):

$$
\beta_{e}=\frac{r_{\text {planck }}}{r_{\text {elec }}}=\frac{r_{\text {planck }}(1 \mathrm{sec})}{r_{\text {elec }}(1 \mathrm{sec})}=\frac{v_{?}}{v_{?}}=\frac{v_{\beta_{e}}}{c}
$$

And solving for $v_{\beta_{e}}$ :

$$
v_{\beta_{e}}=1.26 \times 10^{-14} \mathrm{~m} / \mathrm{sec}
$$

so that 


$$
\beta_{e}=\frac{1.26 \times 10^{-14} \mathrm{~m} / \mathrm{sec}}{c}
$$

As opposed to

$$
\alpha=\frac{2.19 \times 10^{6} \mathrm{~m} / \mathrm{sec}}{c}
$$

for the fine structure constant.

Actually, it should probably be pointed out, at this point, that:

$$
\beta_{e}^{2}=\frac{v^{2}}{c^{2}}=1.75 \times 10^{-45}
$$

which is, in fact, the "accepted" gravitational coupling constant. Also:

$$
\frac{G m_{\text {elec }}}{r_{\text {elec }}}=\left(v_{\beta_{e}}\right)^{2}
$$

which, upon inspection, would seem to represent the largest possible gravitational potential for an electron mass.

And, of course:

$$
\left(m_{\text {planck }} \cdot v_{\beta_{e}} \cdot r_{\text {elec }}\right)=\left(2.18 \times 10^{-8} \mathrm{~kg}\right)\left(1.26 \times 10^{-14} \mathrm{~m} / \mathrm{sec}\right)\left(3.86 \times 10^{-13} \mathrm{~m}\right)=\hbar
$$

"Incidentally", $\left(m_{\text {elec }} \cdot u_{\beta_{e}} \cdot r_{\text {planck }}\right)=\hbar$ also, where $u_{\beta_{e}}$ is the corresponding "phase velocity" as in (Eq. 7).

To continue, though, because $\left(m_{\text {planck }} \cdot v_{\beta_{e}} \cdot r_{\text {elec }}\right)=\hbar$,

$$
\beta_{e}=\frac{\hbar}{\left(m_{\text {planck }}\right)(c)\left(r_{\text {elec }}\right)}
$$

Therefore:

$$
\frac{G m_{\text {prot }} m_{\text {elec }}}{r^{2}}=\frac{m_{\text {prot }}\left(\begin{array}{ll}
u & v
\end{array} r_{\text {planck }}\right.}{r^{2}} \cdot \frac{\hbar}{\left(m_{\text {planck }}\right)(c)\left(r_{\text {elec }}\right)}
$$

And

$$
\frac{r_{\text {planck }}}{m_{\text {planck }}}=\frac{G}{c^{2}}
$$

so

$$
\frac{G m_{\text {prot }} m_{\text {elec }}}{r^{2}}=\frac{(u v)}{r^{2} c^{3}} \cdot \frac{\hbar G m_{\text {prot }}}{r_{\text {elec }}}=\frac{\hbar\left(G m_{\text {prot }}\right)}{\left(r^{2}\right)(c)\left(r_{\text {elec }}\right)}=\frac{\hbar}{r^{2}} \frac{v^{2}}{c}
$$

And $\left(\frac{v^{2}}{c}\right)$ is equal to some different velocity $(v)$, so that:

$$
\frac{G m_{\text {prot }} m_{\text {elec }}}{r^{2}}=\frac{\hbar v}{r^{2}}
$$

where

$$
v=9.63 \times 10^{-34} \mathrm{~m} / \mathrm{sec}
$$


as opposed to (Eq. 4) for the electrostatic force. And note that this velocity is the same as that derived from the factor in blue of (Eq. 2)

The velocity $(v)$ could also be derived as follows:

$$
m_{\text {elec }}=\frac{\hbar \omega_{\text {elec }}}{c^{2}}
$$

And $(u \cdot v)$ is always equal to $\left(c^{2}\right)$, so that:

$$
\begin{gathered}
\left(\frac{r_{\text {planck }}}{r_{\text {prot }}}\right)\left(\frac{\left(m_{\text {elec }}\right)(u v)\left(r_{\text {planck }}\right)}{r^{2}}\right)=\left(\frac{r_{\text {planck }}}{r_{\text {prot }}}\right)\left(\frac{\left(\hbar \omega_{\text {elec }}\right)(u v)\left(r_{\text {planck }}\right)}{c^{2}} \frac{1}{r^{2}}\right) \\
=\left(\frac{r_{\text {planck }}}{r_{\text {prot }}}\right)\left(\frac{\left(\hbar \omega_{\text {elec }}\right)\left(c^{2}\right)\left(r_{\text {planck }}\right)}{c^{2}} \frac{1}{r^{2}}\right)
\end{gathered}
$$

$\left(\frac{r_{\text {planck }}}{r_{\text {prot }}}\right) \cdot\left(\omega_{\text {elec }} r_{\text {planck }}\right)$ is equal to some velocity $v$, so that:

$$
F_{G}=\frac{\hbar v}{r^{2}}
$$

and solving for $v$ results in:

$$
v=9.63 \times 10^{-34} \mathrm{~m} / \mathrm{sec}
$$

as previously calculated.

And finally, if

$$
F_{e}=\frac{\hbar v}{r^{2}}
$$

where $\left(v=(c \cdot \alpha)=2.19 \times 10^{6} \mathrm{~m} / \mathrm{sec}\right)$, and, if the gravitational force is $\left(2.27 \times 10^{39}\right)$ times weaker (in the case of the electron-proton interaction), then it stands to reason that the gravitational force between the two should be:

$$
F_{G}=\frac{\hbar v}{r^{2}}=\frac{\hbar \frac{2.19 \times 10^{6} \mathrm{~m} / \mathrm{sec}}{2.27 \times 10^{39}}}{r^{2}}=\frac{(\hbar)\left(9.63 \times 10^{-34} \mathrm{~m} / \mathrm{sec}\right)}{r^{2}}
$$

again, as previously calculated.

$\left(9.63 \times 10^{-34} \mathrm{~m} / \mathrm{sec}\right)$ is a tiny velocity. How tiny is it? It should be the smallest "force-induced quantum state" velocity possible, for if the gravitational force is the weakest of the four forces, the smallest "induced" velocity would have to be gravitational; likewise, the smallest quantum state gravitational potential $\left(\frac{G m_{\text {elec }}}{r}=v^{2}\right)$ would have to be produced, in this case, by the smallest mass (the electron).

(Eq. 10), then, clearly indicates the first of the double $\mathrm{h}$ bars:

$$
F_{G}=\frac{\hbar v}{r^{2}}
$$

And because it describes the smallest gravitational field, the (Eq. 6) form of the new gravitational field equation shall be used to derive the second ( $\hbar)$. Hence:

$$
\left(\frac{\left(r_{\text {planc }}\right)}{r_{\text {elec }}}\right) \cdot \frac{\left(m_{\text {prot }}(u v) r_{\text {planck }}\right.}{r^{2}}=\frac{G m_{\text {elec }} m_{\text {prot }}}{r^{2}}
$$

In this case, the dimension in question is the reduced wavelength $\left(r_{\lambda}\right)$, for which:

$$
\left(m_{\text {prot }} \cdot\left(9.63 \times 10^{-34} \mathrm{~m} / \mathrm{sec}\right) \cdot r_{\lambda}\right)=\hbar
$$


And since $\left(v=9.63 \times 10^{-34} \mathrm{~m} / \mathrm{sec}\right)$ is so tiny, $\left(r_{\lambda}\right)$ must be enormous.

It should help if one were to imagine a universe consisting of a single proton mass and a single electron mass, and, ignoring electrical charge, to focus only on the gravitational field produced by the electron mass. Remembering that Galileo proved that all masses fall at the same rate in a given gravitational field, this "imaginary" universe could, conceivably, be constructed of one electron mass and one cannonball. What is sought here, however, is the "quantum state," where $\left(m_{\text {prot }} \cdot v \cdot r_{\lambda}\right)=\hbar$. It is fitting to think in these terms, for, after all, our own universe, at one time, consisted of a huge, but equal number of protons and electrons.

Now, if one were to think of the electron's being located at the origin of some coordinate system, and then move the proton further and further and further away...until it were located exactly halfway to the edge of this imaginary universe, it would constitute a gravitational hydrogen atom, of sorts, whose orbital diameter would reach all the way to the edge of this imaginary universe. Now, solving for $\left(r_{\lambda}\right)$ in (Eq. 13), and giving it the designation $\left(\beta_{0}\right)$ :

$$
r_{\lambda}=\beta_{0}=\frac{\hbar}{\left(1.67 \times 10^{-27} \mathrm{~kg}\right)\left(9.63 \times 10^{-34} \mathrm{~m} / \mathrm{sec}\right)}=6.53 \times 10^{25} \mathrm{~m}
$$

A radius of $\left(6.53 \times 10^{25} \mathrm{~m}\right)$ would mean a diameter of $\left(1.31 \times 10^{26} \mathrm{~m}\right)$. Therefore, the distance to the edge of this universe would have to be:

$$
\frac{1.31 \times 10^{26} \mathrm{~m}}{3.0 \times 10^{8} \mathrm{~m} / \mathrm{sec}}=4.36 \times 10^{17} \text { light seconds }
$$

or:

$$
\left(4.36 \times 10^{17} \text { light sec }\right) \cdot \frac{1 \mathrm{~min}}{60 \mathrm{sec}} \cdot \frac{1 \text { hour }}{60 \mathrm{~min}} \cdot \frac{1 \text { day }}{24 \text { hours }} \cdot \frac{1 \text { year }}{365.25 \text { days }}=13.81 \times 10^{9} \text { light years. } \quad 15
$$

i.e. $\left(\beta_{0}\right.$ times 2$)$ is equal to 13.81 billion light years.

"How can this be," one would ask? "13.81 billion years?" This is the approximate age of the "real"the "existing"—universe. But the actual universe is expanding-it's age and size, continually changing. In addition, $\left(\beta_{0}\right)$, it would seem, is dependent upon a series of universal "constants"; although it must be remembered that these constants were derived during the current epoch. Also, it would appear that, the present situation represents the lowest possible energy state; in which case (Eq. 13) should actually look like this:

$\left(m_{\text {prot }} \cdot\left(\frac{9.63 \times 10^{-34} \mathrm{~m} / \mathrm{sec}}{n}\right) \cdot\left(n^{2}\right) r_{\lambda}\right)=(n) \hbar$

with the quantum number $(n)$ as in the case of the classical hydrogen atom. The next highest energy state, therefore, would be the one at which $(n=2)$, and $\left(\left(n^{2}\right) r_{\lambda}=\left(2^{2}\right) \beta_{0}=2.61 \times 10^{26}\right.$ meters $)$, i.e. about 40 billion years from now. But then, that's just conjecture.

Our present dilemma involves a situation in which (simultaneously),

$$
\begin{gathered}
v^{2}=\frac{G m_{\text {elec }}}{\beta_{0}}=\left(9.63 \times 10^{-34} \mathrm{~m} / \mathrm{sec}\right)^{2} \\
u=\frac{c^{2}}{v} \\
\left(m_{\text {prot }}(v) \beta_{0}=\hbar\right),
\end{gathered}
$$

and

$$
\left(\frac{\left(r_{\text {planck }}\right)}{r_{\text {elec }}}\right) \cdot\left(m_{\text {prot }}(u) r_{\text {planck }}=\hbar\right)
$$


Is this some remarkable coincidence? If so, what is the significance of (Eq. 15)?

In all honesty, no definitive answer shall be found herein; nevertheless, a particle traveling for $\left(\frac{\beta_{0}}{c}=\frac{13.81}{2}\right)$ billion years $\left(2.18 \times 10^{17}\right.$ seconds $)$ at $\left(9.63 \times 10^{-34} \mathrm{~m} / \mathrm{sec}\right)$ would travel $\left(2.11 \times 10^{-16}\right.$ meters $)$, or exactly one proton reduced wavelength.

To bring up another remarkable coincidence, one proton mass corresponds to an oscillation frequency of $1.42 \times 10^{24}$ radians per second (i.e. $\left.m c^{2}=\hbar \omega\right)$; in this case, $\left(r_{\lambda}=\beta_{0}\right)$ is $6.53 \times 10^{25}$ meters. $\left(r_{\lambda}=\frac{1}{k}\right)$, so that $\left(r_{\lambda} \cdot \omega=\frac{\omega}{k}\right)$, which is the phase velocity $(u)$, and, as previously stated, $\left(\frac{m_{2} u^{2}}{r_{\lambda}}=\frac{c^{4}}{G}\right)$, so that:

$\frac{m_{\text {elec }} u^{2}}{r}=\frac{\left(9.11 \times 10^{-31} \mathrm{~kg}\right)\left(\left(6.53 \times 10^{25} \mathrm{~m}\right)\left(1.42 \times 10^{24} \mathrm{rad} / \mathrm{sec}\right)\right)^{2}}{6.53 \times 10^{25} \mathrm{~m}}=1.21 \times 10^{44}$ newtons $=\frac{c^{4}}{G}$

the Planck force.

Now if $u=\left(r_{\lambda} \omega\right)$ and $\left(\omega=\frac{m c^{2}}{\hbar}\right)$, then $\left(u=\frac{r_{\lambda} M_{\text {prot }} c^{2}}{\hbar}\right)$ and $\left(v=\frac{c^{2}}{u}=\frac{c^{2} \hbar}{r_{\lambda} m_{\text {prot }} c^{2}}=\frac{\hbar}{r_{\lambda} m_{\text {prot }}}\right.$, and $v^{2}=\frac{\hbar \hbar}{r_{\lambda}^{2}\left(m_{\text {prot }}\right)^{2}}$, so that:

$$
F_{G}=\frac{m_{\text {prot }} v^{2}}{r_{\lambda}}=\frac{\hbar \hbar}{r_{\lambda}^{3} m_{\text {prot }}}
$$

It was shown earlier that

$$
\frac{c^{2} \times 10^{-7} q_{\text {elec }} q_{\text {prot }}}{\alpha_{0}^{2}}=\frac{(-1) \hbar(+1) \hbar}{m_{\text {elec }} \alpha_{0}^{3}}
$$

for the electrostatic force, and that

$$
\frac{c^{2} \times 10^{-7} q q}{r^{2}}=\frac{( \pm n) \hbar( \pm n) \hbar}{r^{2} m_{\text {elec }} \alpha_{0}}
$$

for any $(r)$ and any integer $(n)$. It also happens that,

$$
\frac{G m_{\text {elec }} m_{\text {prot }}}{\beta_{0}^{2}}=\frac{(1) \hbar(1) \hbar}{m_{\text {prot }} \beta_{0}^{3}}
$$

and

$$
\frac{G m_{\text {elec }} m_{\text {prot }}}{r^{2}}=\frac{(1) \hbar(1) \hbar}{r^{2} m_{\text {prot }} \beta_{0}}
$$

where, $\left(\beta_{0}=6.54 \times 10^{25} \mathrm{~m}\right)$, as in (Eq. 14).

And, of course,

$$
\frac{G(2) m_{\text {elec }}(2) m_{\text {prot }}}{r^{2}}=\frac{(2) \hbar(2) \hbar}{r^{2} m_{\text {prot }} \beta_{0}}
$$

leading to

$$
\frac{G\left(n_{e}\right) m_{\text {elec }}\left(n_{p}\right) m_{\text {prot }}}{r^{2}}=\frac{(n) \hbar(n) \hbar}{r^{2} m_{\text {prot }} \beta_{0}}
$$

for any $(r)$ and any integers $\left(n_{e}\right)$ and $\left(n_{p}\right)$. 
In view of the fact that the real universe consisted, early on, of a huge but equal number of electrons and protons, (Eq. 16) would represent each and every possible integer product of these gravitational masses, or products of sums thereof.

On the macro-scale, of course, it is possible to approximate. For instance, if it were necessary to find the gravitational acceleration, at the earth's surface, of an electron, or a cannon ball, or even a human being, one need only to approximate the integer number of proton equivalent masses contained in the earth's total mass. For instance:

$$
n=n_{\text {earth }}=\frac{m_{\text {earth }}}{m_{\text {prot }}}=\frac{6.0 \times 10^{24} \mathrm{~kg}}{1.67 \times 10^{-27} \mathrm{~kg}}=3.59 \times 10^{51}
$$

or there about. The earth's radius is about $\left(r=6.4 \times 10^{6}\right.$ meters), in which case, (Eq. 16) would result in the following:

$$
\frac{G m_{\text {earth }}}{(r)^{2}}=\frac{\left(3.59 \times 10^{51}\right) \hbar(1) \hbar}{(r)^{2} m_{\text {elec }} m_{\text {prot }} \beta_{0}}=9.8 \mathrm{~m} / \mathrm{sec}^{2}
$$

